\title{
Erratum to: Fifth metacarpal neck fractures treated with soft wrap/buddy taping compared to reduction and casting: results of a prospective, multicenter, randomized trial
}

Jan van Aaken ${ }^{1}\left(\mathbb{D} \cdot\right.$ Cesare Fusetti $^{2} \cdot$ Stefano Lucchina $^{3} \cdot$ Stefania Brunetti $^{2} \cdot$ Jean-Yves Beaulieu ${ }^{1}$ - Angèle Gayet-Ageron ${ }^{1}$ - Kathryn Hanna ${ }^{4}$ - Alexander Y. Shin ${ }^{5}$. Eric Hofmeister ${ }^{4}$

Published online: 7 September 2016

(c) Springer-Verlag Berlin Heidelberg 2016

Erratum to: Arch Orthop Trauma Surg (2016)

\section{6:135-142}

DOI 10.1007/s00402-015-2361-0

In the original article, one of the co-author's (Stefano Lucchina) family name has been published incorrectly. The correct family name should be Lucchina.

The online version of the original article can be found under doi:10.1007/s00402-015-2361-0.

$\triangle$ Jan van Aaken

Jan.vanaaken@hcuge.ch

1 University Hospital of Geneva (HUG), Rue Gabrielle-PerretGentil 4, 1211 Geneva, Switzerland

2 Ente Ospedaliero Cantonale (EOC), Bellinzona, Switzerland

3 Ente Ospedaliero Cantonale (EOC), Locarno, Switzerland

4 Naval Medical Center in San Diego, San Diego, USA

5 Mayo Clinic, Rochester, USA 